\title{
Aspiration and Injection of the Knee Joint: Approach Portal
}

\author{
Robert J. Douglas, MD \\ Medical Division, Sportsmed.SA, Stepney SA, Australia
}

\begin{abstract}
Aspiration and injection of the knee joint is a commonly performed medical procedure. Injection of corticosteroid for the treatment of osteoarthritis is the most common reason for knee joint injection, and is performed as an office procedure. Debate exists among practitioners as to the 'best' approach portal for knee injection. This paper examines the various approach portals for injection and/or aspiration of the knee joint, as well as the accuracy of each approach. Searches were made of electronic databases, and appropriate papers were identified and hand-searched. Although there is some evidence that particular approach portals may be more efficacious in the presence of specific knee joint pathologies, generally, in experienced hands, it is of no clinical consequence as to which approach portal is utilised for aspiration or injection of the knee joint. No approach portal is $100 \%$ accurate, and the accuracy of injection of the knee joint may be enhanced by the use of techniques such as ultrasound. Practitioners are reminded that they should continuously refine and practice their preferred technique. Knee joint aspiration and injection is a common, simple, and generally safe office procedure.
\end{abstract}

Keywords: Knee joint, Osteoarthritis, Injections, Intra-articular

\section{Introduction}

Aspiration of the knee joint may be performed for the diagnosis of an unexplained effusion, or the evacuation of a painful effu$\operatorname{sion}^{1)}$. Injection of the knee can be undertaken for radiological investigation of the $\mathrm{knee}^{2)}$, for the injection of corticosteroid into a joint suffering from a non-infectious inflammatory process ${ }^{3)}$, or for the injection of viscosupplementation ${ }^{4)}$. Of these indications for aspiration or injection of the knee joint, the most common is the injection of corticosteroid in cases of osteoarthritis (OA) of the knee, and is performed as an office procedure.

A recent article ${ }^{5)}$ examined the choice and utilisation of corti-

Received July 8, 2013; Revised (1st) October 7, 2013;

(2nd) December 1, 2013; Accepted December 5, 2013

Correspondence to: Robert J. Douglas, MD

Medical Division, Sportsmed.SA, 32 Payneham Road, Stepney SA 5069, Australia

Tel: +61-4-0888-1569, Fax: +61-8-8342-5118

E-mail: rabs01@hotmail.com

This is an Open Access article distributed under the terms of the Creative Commons Attribution Non-Commercial License (http://creativecommons.org/licenses/by-nc/3.0/) which permits unrestricted non-commercial use, distribution, and reproduction in any medium, provided the original work is properly cited. costeroid agents for injection into the osteoarthritic knee, and suggested that it is traditional clinical Orthopaedic Surgery and Rheumatology teaching which determines the likelihood of utilisation of one corticosteroid (and dosing regimen) over another. Similarly, disagreement also exists among practitioners as to the 'best' approach portal for knee aspiration and injection. This article examines the different approach portals for aspiration or injection of the knee joint with the aim of: describing the available approach portals for aspiration or injection of the knee joint; determining if indeed there is a 'best' approach portal; determining whether the 'best' approach portal for knee joint aspiration is the same as that for injection; and examining the possible problems encountered with each approach portal.

Finally, the paper makes recommendations for improvement in technique by medical practitioners.

\section{Approaches to Aspiration or Injection of the Knee Joint}

The first reported use of intra-articular (IA) corticosteroid in knee OA was by Hollander ${ }^{6}$ in 1953, and the first clinical trial of IA steroid use for knee OA was reported by Miller et al. ${ }^{7)}$ in 1958. Six major portals of approach to the knee joint for its aspiration 
Table 1. Reported Accuracy for Each Major Knee Injection Technique

\begin{tabular}{|c|c|c|c|}
\hline Approach & Author & Success rate $(\%)$ & Comments \\
\hline \multirow[t]{3}{*}{ Laternal midpatellar } & Esenyal et al. ${ }^{2)}$ & 76 & Accuracy may be proportional to severity of knee osteoarthritis \\
\hline & Jackson et al. ${ }^{11)}$ & 93 & May be uncomfortable or painful for patient \\
\hline & Toda and Tsukimura ${ }^{9)}$ & $55-86^{\text {a) }}$ & \\
\hline \multirow[t]{2}{*}{ Laternal midpatellar } & Esenyal et al. ${ }^{2)}$ & 56 & May allow easier access if patient has knee osteoarthritis \\
\hline & & & May be uncomfortable or painful for patient \\
\hline Superolateral & Hermans et al. ${ }^{17)}$ & $91^{\mathrm{b})}$ & May be painful if collides with superior pole of patella \\
\hline Superomedial & Wind and Smolinski ${ }^{13)}$ & 93 & May damage patella chondral cartilage \\
\hline \multirow[t]{2}{*}{ Anterolateral } & Esenyal et al. ${ }^{2)}$ & 85 & \\
\hline & Jackson et al. ${ }^{11)}$ & 71 & Useful when knee cannot be extended \\
\hline \multirow[t]{3}{*}{ Anteromedial } & Esenyal et al. ${ }^{2)}$ & 73 & Little pain or discomfort to patient \\
\hline & Jackson et al. ${ }^{11)}$ & 75 & May be difficult to aspirate knee effusion \\
\hline & Toda and Tsukimura ${ }^{9)}$ & 55 & \\
\hline
\end{tabular}

${ }^{\text {a) }}$ Varied according to Kellgren-Lawrence rating, ${ }^{\text {b) }}$ Pooled data.

or injection have been described: the lateral and medial midpatellar (MMP) and patellofemoral; the superolateral and superomedial; and anterolateral and anteromedial (inferolateral and inferomedial) approaches. A recent on-line video series by GarciaRodrigue ${ }^{8)}$ demonstrates injection of the knee joint by several of these approaches.

Each portal of approach has its own advantages and disadvantages, and each of the major techniques is discussed below. Table 1 provides a summary of the efficacy of each of the major injection approaches.

\section{Lateral and Medial Mid-Patellar and Patello-Femoral Approaches}

The traditional approach to knee injection accesses the patellofemoral joint ${ }^{1}$ (Fig. 1). This technique is performed with the knee in extension. The patella is pulled medially or laterally and a needle is advanced under the patella. The lateral midpatellar (LMP) approach is the most commonly used ${ }^{2}$. The patellofemoral joint, via the LMP and MMP approaches can then be used for joint aspiration and/or injection. When performing a procedure via the LMP approach, the needle is directed at a $45^{\circ}$ angle towards the middle of the medial aspect of the joint. Injection via the MMP approach is undertaken with the needle entering the medial aspect of the knee joint under the middle of the patella (midpole), and is directed towards the lateral patellar midpole ${ }^{3)}$. It is reported that most (US) rheumatologists prefer the medial approach with the knee extended and the patient lying down because the lateral patellofemoral cleft is narrower, and the joint capsule is tougher laterally than medially ${ }^{3}$.

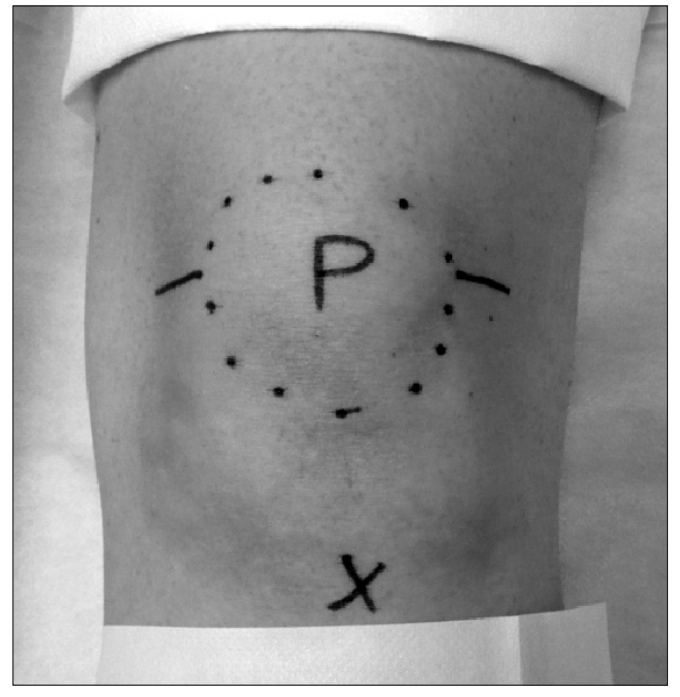

Fig. 1. Photograph of left knee. Patella (P) is circled. The tibial tuberosity is marked with a cross. Lines indicate the access points for medial and lateral midpatellar approaches to aspiration or injection of the knee joint.

A survey of US radiologists by Shortt et al. ${ }^{4)}$ found that the lateral patellofemoral approach was the most commonly utilized technique for knee arthrography (41/64 respondents, 64\%), mirroring previous work by Freiberger ${ }^{1)}$ and Esenyal et al..$^{2)}$ The medial patellofemoral approach was preferentially utilized by only $16 / 64$ respondents (25\%). Reasons for preferring a medial patellofemoral approach included difficulty with lateral access secondary to patellofemoral OA. Esenyal et al. ${ }^{2)}$ reported in their study of knee joint injection that the MMP approach is the least accurate of all tested IA approaches (56\%). Toda and Tsukimura ${ }^{9)}$ found that 
they achieved an accuracy of 55\%-86\% for the LMP approach and that accuracy was directly proportional to severity of radiographic OA assessment (Kellgren-Lawrence grading) ${ }^{10}$. Esenyal et al. ${ }^{2)}$ reported an accuracy of $76 \%$, and Jackson et al. ${ }^{11)}$ reported an accuracy of $93 \%$ (74/80) for the LMP approach. Aspiration or injection via the LMP or MMP approaches has been reported to be uncomfortable for patients, and can be painful if access is difficult. These approaches can be difficult when patients tense the thigh extensors; in the obese; and in patients with patellofemoral arthrosis ${ }^{12}$. Wind and Smolinski ${ }^{13)}$ have suggested that, as lateral joint line injections (ie LMP) may not be reliable for routine injections of low volumes of fluid into the knee joint as it results in good IA delivery less than half the time with a high incidence of soft-tissue infiltration, a superomedial or superolateral approach should be used instead.

\section{Superolateral and Superomedial Approaches}

Although the usual site of entry to the knee joint is via either an LMP or MMP approach, an approach lateral and superior to the patella can be used, especially if there is a large effusion in the suprapatellar bursa ${ }^{14)}$ (Fig. 2). This technique is performed with the patient supine and the knee extended. Zuber ${ }^{15)}$ states that this approach provides the most direct access to the synovium. Sher ${ }^{16)}$ advocates the use of the superolateral approach (with the knee extended), for aspiration. This technique aims for the suprapatellar pouch, and allows the needle to pass underneath the articular surface of the patella. Hermans et al. ${ }^{17)}$ determined that the superolateral approach results in the highest pooled accuracy rate of $91 \%$ (95\% CI 84\%-99\%), although this approach still results

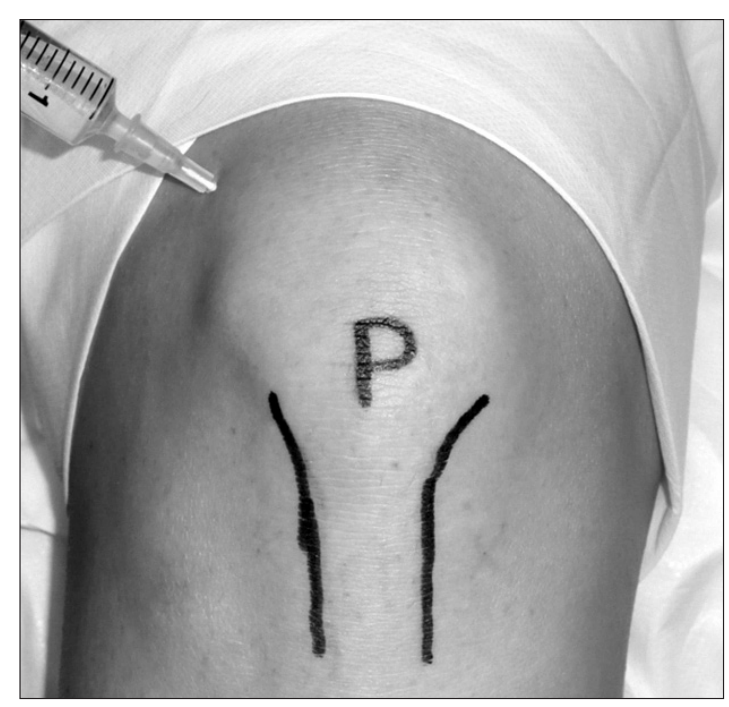

Fig. 2. Photograph of right knee demonstrating the superolateral approach to aspiration or injection of the knee via the suprapatellar bursa. in a substantial amount of extra-articular needle placements. In their study observing the effect of combined joint lavage and corticosteroid injection upon ninety-eight patients with symptomatic knee OA, Ravaud et al. ${ }^{18)}$ performed aspiration and injection of the joint via the superolateral approach. More recently, Ucar et al. ${ }^{19)}$ used the same approach in their study of hyaluronic acid injection into the osteoarthritic knee. The superomedial approach is poorly studied with only a single trial, and a reported accuracy of $93 \%{ }^{13)}$.

Accidental collision of the needle tip with the superolateral or superomedial poles of the patella during attempted aspiration or injection of the knee joint have led to problems with pain, as well as damage to the chondral cartilage of the patella. These problems may be avoided by using the inferolateral or inferomedial poles of the patella (ie an anterior or arthroscopic approach) as a needle insertion site ${ }^{20)}$.

\section{Anterolateral and Anteromedial (Arthroscopic) Approaches}

The anterior (or infrapatellar) approaches (Fig. 3) are an alternative to the classic patellofemoral approaches, and are used less often for knee joint injection and aspiration ${ }^{14,21)}$. Shortt et al. ${ }^{4)}$ reported that only 7/64 (11\%) of surveyed North American musculoskeletal radiologists preferentially utilised an arthroscopic approach for injection of the knee joint. Anterior techniques utilise an entry approach analogous to the portals used for knee arthroscopy ${ }^{12,21}$. These approaches are useful when the knee cannot be extended, or when there is only a minimal amount of fluid in the knee joint ${ }^{14}$. With the knee flexed, the needle is introduced

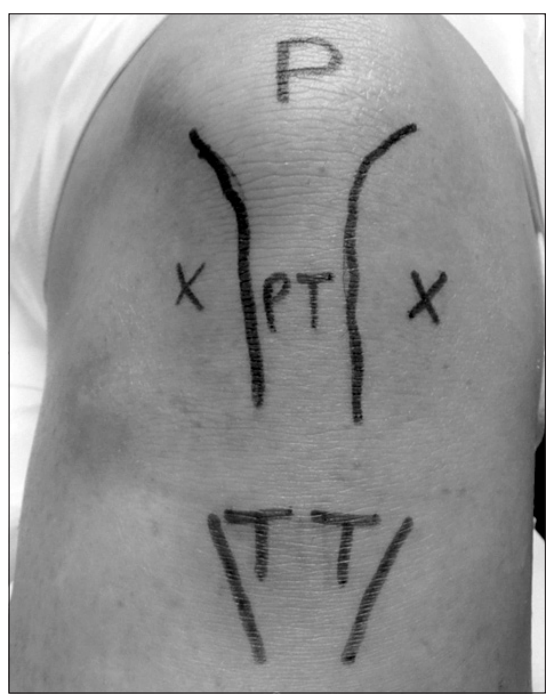

Fig. 3. Photograph of left knee. Crosses indicate the anteromedial and anterolateral approaches to aspiration or injection of the knee joint. P: patella, PT: patellar tendon, TT: tibial tuberosity. 
either lateral or medial to the patella tendon ${ }^{15}$, and is directed upwards towards the femoral notch ${ }^{2,22}$. There is no need to manipulate the patella ${ }^{11)}$. These approaches traverse only Hoffa's fat pad and they avoid the extensor mechanism and major blood vessels. With correct technique, the anterior horns of the menisci and the transverse patellar ligaments are easily avoided ${ }^{12,21}$. Jackson et al. ${ }^{11}$ examined the accuracy of knee joint injection by a single orthopaedic surgeon using these approach portals. They reported an accuracy of $71 \%$ (57/80 attempts) for the anterolateral approach and $75 \%$ (60/80 attempts) for an anteromedial approach (compared to the $93 \%$ accuracy for LMP approach). Toda and Tsukimura ${ }^{9}$ reported an accuracy of only 55\% (6/11 attempts) for a seated anteromedial approach. Esenyal et al. ${ }^{2)}$ compared the accuracy of the anterolateral, anteromedial, LMP and MMP approaches. They determined that the anterolateral approach was the most accurate (85\%) but that this was not statistically significant compared to the anteromedial (73\%) and LMP (76\%) approaches. It is reported that the arthroscopic approaches involve little pain or discomfort ${ }^{12)}$. The major problem with these approaches has been reported to be difficulty in obtaining fluid from an affected joint ${ }^{14)}$.

\section{Other Approaches}

Waddell et al. ${ }^{22)}$ used a technique that placed the knee into $30^{\circ}-40^{\circ}$ flexion, and inserted the needle $1-1.5 \mathrm{~cm}$ proximal from the anterolateral arthroscopy port, aiming the needle towards the anterior contact point between the femoral condyle and the tibial plateau. They reported an accuracy of $100 \%$. Toda and Tsukimura ${ }^{9)}$ reported an accuracy of $86 \%$ utilising a modified Waddell's approach, which moved the knee into $30^{\circ}$ flexion with concurrent traction upon the ankle, and injecting the knee from a point $1-1.5 \mathrm{~cm}$ above the anteromedial injection site, aiming towards the anterior contact point between the femoral condyle and the tibial plateau ${ }^{17}$. However, both papers are the sole reports of the use of these techniques. Cohn and Shapiro ${ }^{20)}$ described an anterior approach that utilized injection through the patellar tendon. However, despite the ease of approach, they determined that the technique led to adverse outcomes related to injection into Hoffa's fat pad, or into the anterior cruciate ligament. Cardone and Tallia ${ }^{3)}$ have described an anterior approach, which aims the needle parallel to the tibial plateau. However, this technique risks meniscal damage by the needle.

Sambrook et al. ${ }^{23)}$ described an interesting variation of access of corticosteroid to the IA space. They used a peripatellar injection of corticosteroid, as they considered IA injection of corticosteroid to be of uncertain efficacy and duration of effect. Their technique provided results that were not significantly different from that achieved with their control group, who had received standard IA corticosteroid injection.

\section{Improving the Accuracy of Injection}

Numerous studies have shown that a variable proportion of IA injections are placed correctly into the target joint space s,9,11,13,24,25) $^{2}$ and the injection of IA corticosteroid has been shown to be more effective when it is correctly placed in the joint ${ }^{25,26)}$. The inaccurate placement of injecting needles can result in damage to IA structures secondary to soft tissue infiltration by corticosteroid $^{13,25)}$. Clinical experience has shown that IA injection of the knee joint is more painful when the tip of the needle is in Hoffa's fat pad. This is also the most common ectopic injection site for missed IA injection ${ }^{2)}$. Chavez-Chang et al. ${ }^{27)}$ have suggested that the length of the needle used for IA injection may influence IA accuracy, as the standard $3.8 \mathrm{~cm}$ needle may be too short to reach the synovial space overlying the medial femoral condyle. A review by Berkoff et al. ${ }^{28)}$ has suggested that the accuracy of IA injection may be improved by the utilization of ultrasound guidance, with an accuracy of $95.8 \%$ (versus $77.8 \%$ for anatomical guidance [p<0.001, OR 6.4 \{95\% CI: 2.9-14\}]). Park et al. ${ }^{29)}$ reported an accuracy raging from $95 \%$ (medial midpatellar approach) to $100 \%$ (superolateral approach) for the use of ultrasound guidance using a long axis in plane technique. Maricar et al. ${ }^{30)}$ stated that injection under ultrasound guidance may improve the likelihood of response to IA corticosteroid injection, and Sibbitt et al. ${ }^{31)}$ reported that ultrasound-guided aspiration and injection of the knee resulted in improved aspiration success, greater synovial fluid yield, more complete joint decompression, and improved clinical outcomes.

It has been suggested that accuracy of IA knee injection can be further improved by the use of mini-air arthrography ${ }^{32}$, or by the combined use of air injection into the target joint with concurrent use of an ultrasound probe ${ }^{33,34)}$. Despite their presumed efficacy, these two techniques would appear to be beyond the usual expertise and resources of those medical practitioners that commonly aspirate and/or inject the knee joint.

\section{Conclusions}

Examination of the literature investigating the various approach portals for knee joint aspiration and/or injection determined that there are six major techniques. There is no evidence for an optimal or 'best' technique, although there is some suggestion 
that one or two techniques may be inferior to others. Particular portals of approach may be more efficacious for particular knee joint pathologies, for example a medial patellofemoral approach may be easier to perform in a patient suffering from patellofemoral subluxation. Available evidence suggests that, in the hands of experienced practitioners, it is of no clinical consequence which approach portal is used for the anatomical guidance of aspiration or injection of the knee joint-any of the described approaches may be utilised depending upon the experience and preference of the practitioner. However, practitioners must bear in mind that no approach has $100 \%$ efficacy for either aspiration or injection of the knee joint, and incorrect use of any of the techniques may cause pain, or damage internal knee structures.

Although techniques are available that can enhance the accuracy of IA aspiration or injection, access to the required equipment and familiarity with its use may be beyond the resources, capabilities, and expertise of most practitioners and their practices. This paper demonstrates that the traditional approaches to aspiration or injection of the knee are easily performed and are generally safe, but also highlights the need for practitioners to continuously refine and practice their preferred aspiration and injection technique of the knee joint.

\section{Conflict of Interest}

No potential conflict of interest relevant to this article was reported.

\section{Acknowledgements}

My thanks go to Dr. Tonia Mezzini of Clinic 275, Royal Adelaide Hospital, for her assistance with the preparation of the manuscript, and to Miss Brooke Smith of Sportsmed SA, for her assistance with the preparation and posing of knee photographs.

\section{References}

1. Freiberger RH. Technique of knee arthrography. In: Freiberger RH, Kaye JJ, Spiller J, eds. Arthrography. New York: Appleton-Century-Crofts; 1979. p5-30.

2. Esenyel C, Demirhan M, Esenyel M, Sonmez M, Kahraman S, Senel B, Ozdes T. Comparison of four different intra-articular injection sites in the knee: a cadaver study. Knee Surg Sports Traumatol Arthrosc. 2007;15:573-7.

3. Cardone DA, Tallia AF. Diagnostic and therapeutic injection of the hip and knee. Am Fam Physician. 2003;67:2147-52.
4. Shortt CP, Morrison WB, Roberts CC, Deely DM, Gopez AG, Zoga AC. Shoulder, hip, and knee arthrography needle placement using fluoroscopic guidance: practice patterns of musculoskeletal radiologists in North America. Skeletal Radiol. 2009;38:377-85.

5. Douglas RJ. Corticosteroid injection into the osteoarthritic knee: drug selection, dose, and injection frequency. Int J Clin Pract. 2012;66:699-704.

6. Hollander JL. Intra-articular hydrocortisone in arthritis and allied conditions; a summary of two years' clinical experience. J Bone Joint Surg Am. 1953;35:983-90.

7. Miller JH, White J, Norton TH. The value of intra-articular injections in osteoarthritis of the knee. J Bone Joint Surg Br. 1958;40:636-43.

8. Garcia-Rodriguez JA. Intra-articular knee injections: procedures and assessments video series. Can Fam Physician. 2013;59:377.

9. Toda Y, Tsukimura N. A comparison of intra-articular hyaluronan injection accuracy rates between three approaches based on radiographic severity of knee osteoarthritis. Osteoarthritis Cartilage. 2008;16:980-5.

10. Kellgren JH, Lawrence JS. Radiological assessment of osteoarthrosis. Ann Rheum Dis. 1957;16:494-502.

11. Jackson DW, Evans NA, Thomas BM. Accuracy of needle placement into the intra-articular space of the knee. J Bone Joint Surg Am. 2002;84:1522-7.

12. Zurlo JV, Towers JD, Golla S. Anterior approach for knee arthrography. Skeletal Radiol. 2001;30:354-6.

13. Wind WM Jr, Smolinski RJ. Reliability of common knee injection sites with low-volume injections. J Arthroplasty. 2004;19:858-61.

14. Neustadt DH. Intra-articular injections for osteoarthritis of the knee. Cleve Clin J Med. 2006;73:897-8, 901-4, 906-11.

15. Zuber TJ. Knee joint aspiration and injection. Am Fam Physician. 2002;66:1497-500.

16. Sher D. Knee Injectio techniques [Internet]. New South Wales; Medical Observer; 2010 [cited 2010 Jul 23]. Available from: http://www.medicalobserver.com.au/.

17. Hermans J, Bierma-Zeinstra SM, Bos PK, Verhaar JA, Reijman $M$. The most accurate approach for intra-articular needle placement in the knee joint: a systematic review. Semin Arthritis Rheum. 2011;41:106-15.

18. Ravaud P, Moulinier L, Giraudeau B, Ayral X, Guerin C, Noel E, Thomas P, Fautrel B, Mazieres B, Dougados M. Effects of joint lavage and steroid injection in patients with osteoarthritis of the knee: results of a multicenter, randomized, 
controlled trial. Arthritis Rheum. 1999;42:475-82.

19. Ucar D, Diracoglu D, Suleyman T, Capan N. Intra-articular hyaluronic Acid as treatment in elderly and middleaged patients with knee osteoarthritis. Open Rheumatol J. 2013;7:38-41.

20. Cohn BT, Shapiro PS. An effective technique for corticosteroid injection into the knee joint. Orthop Rev. 1993;22:13412.

21. Moser T, Moussaoui A, Dupuis M, Douzal V, Dosch JC. Anterior approach for knee arthrography: tolerance evaluation and comparison of two routes. Radiology. 2008;246:193-7.

22. Waddell D, Estey D, DeWayne C, Bricker P, Marsala A. Viscosupplementation under fluoroscopic control. Am J Med Sports 2001;4:237-41.

23. Sambrook PN, Champion GD, Browne CD, Cairns D, Cohen ML, Day RO, Graham S, Handel M, Jaworski R, Kempler S, et al. Corticosteroid injection for osteoarthritis of the knee: peripatellar compared to intra-articular route. Clin Exp Rheumatol. 1989;7:609-13.

24. Eustace JA, Brophy DP, Gibney RP, Bresnihan B, FitzGerald O. Comparison of the accuracy of steroid placement with clinical outcome in patients with shoulder symptoms. Ann Rheum Dis. 1997;56:59-63.

25. Jones A, Regan M, Ledingham J, Pattrick M, Manhire A, Doherty M. Importance of placement of intra-articular steroid injections. BMJ. 1993;307:1329-30.

26. Gaffney K, Ledingham J, Perry JD. Intra-articular triamcinolone hexacetonide in knee osteoarthritis: factors influencing the clinical response. Ann Rheum Dis. 1995;54:379-81.
27. Chavez-Chiang CE, Sibbitt WL Jr, Band PA, Chavez-Chiang NR, Delea SL, Bankhurst AD. The highly accurate anteriolateral portal for injecting the knee. Sports Med Arthrosc Rehabil Ther Technol. 2011;3:6.

28. Berkoff DJ, Miller LE, Block JE. Clinical utility of ultrasound guidance for intra-articular knee injections: a review. Clin Interv Aging. 2012;7:89-95.

29. Park KD, Ahn JK, Lee SC, Lee J, Kim J, Park Y. Comparison of ultrasound-guided intra-articular injections by long axis in plane approach on three different sites of the knee. Am J Phys Med Rehabil. 2013;92:990-8.

30. Maricar N, Callaghan MJ, Felson DT, O’Neill TW. Predictors of response to intra-articular steroid injections in knee osteoarthritis: a systematic review. Rheumatology (Oxford). 2013;52:1022-32.

31. Sibbitt WL Jr, Kettwich LG, Band PA, Chavez-Chiang NR, DeLea SL, Haseler LJ, Bankhurst AD. Does ultrasound guidance improve the outcomes of arthrocentesis and corticosteroid injection of the knee? Scand J Rheumatol. 2012;41:6672.

32. Bliddal $\mathrm{H}$. Placement of intra-articular injections verified by mini air-arthrography. Ann Rheum Dis. 1999;58:641-3.

33. Fredberg U, van Overeem Hansen G, Bolvig L. Placement of intra-articular injections verified by ultrasonography and injected air as contrast medium. Ann Rheum Dis. 2001;60:542.

34. Qvistgaard E, Kristoffersen H, Terslev L, DanneskioldSamsoe B, Torp-Pedersen S, Bliddal H. Guidance by ultrasound of intra-articular injections in the knee and hip joints. Osteoarthritis Cartilage. 2001;9:512-7. 